\title{
APROXIMACIONES A / DESDE LA SEMIÓTICA DE LA ACTUALIDAD
}

\section{APPROACHES FROM / TO THE SEMIOTICS OF THE PRESENT}

\author{
Charo LACALLE ZALDUENDO \\ Universidad Autónoma de Barcelona \\ rosario.lacalle@uab.cat \\ Mario de la TORRE-ESPINOSA \\ Universidad de Granada \\ mariodelatorre@ugr.es
}

\begin{abstract}
Resumen: En esta sección monográfica se reflexiona acerca de la importancia de la semiótica como metodología para abordar fenómenos recientes como la pandemia por la COVID-19, los problemas de representación provocados por el patriarcado o el papel de las redes sociales en la comunicación política contemporánea. Asimismo, se aprovecha para reflexionar acerca de la propia disciplina y su relación con el concepto actualidad, dando como resultado seis contribuciones desarrolladas por especialistas internacionales del campo de la semiótica que iluminan con sus trabajos temas tan diversos como necesarios de reflexionar en nuestra contemporaneidad.
\end{abstract}

Palabras clave: Semiótica. COVID-19. Feminismo. Actualidad. Política.

\begin{abstract}
This monographic section reflects on the importance of semiotics as a methodology for approaching current issues, such as the pandemic caused by COVID19 , the problems of representation generated by patriarchy, or the role of social networks in contemporary political communication. It also represents an opportunity to reflect on the discipline itself and its relationship with the present. The results are six contributions developed by international specialists in the field of semiotics, who discuss about different central topics related to our contemporary world.
\end{abstract}

Keywords: Semiotics. COVID-19. Feminism. Current Topics. Politics.

\section{INTRODUCCIÓN}

En un momento de tanta incertidumbre ante la contingencia de la situación actual, se hace urgente dar sentido a los acontecimientos sobrevenidos. Es por ello que la semiótica se presenta como una disciplina necesaria para reflexionar sobre los hechos acaecidos 
recientemente que han ocasionado una crisis sanitaria y social sin precedente para las generaciones más jóvenes.

Dada esta coyuntura, la Asociación Española de Semiótica (AES) ${ }^{1}$ vio la oportunidad de organizar el Seminario Internacional Semiótica y relatos de la actualidad durante los días 2, 9 y 16 de diciembre de 2020. Con el objetivo de abordar diferentes temáticas desde unos posicionamientos semióticos rigurosos, la actividad, dirigida por Charo Lacalle Zalduendo, José Romera Castillo y Mario de la Torre-Espinosa, contó con un plantel académico de primer nivel que trató en tres mesas redondas aspectos como la pandemia, el género o la actualidad de la disciplina. Se trataba de hacer dialogar a grandes especialistas del ámbito hispánico para demostrar la idoneidad de la semiótica a la hora de desentrañar el significado de los hechos sociales y culturales a los que nos enfrentamos en nuestros días.

Además, con este monográfico se rinde homenaje al vigésimo aniversario del Centro de Investigación de Semiótica Literaria, Teatral y Nuevas Tecnologías SELITEN@T², institución académica que, con la atenta dirección del profesor y nombre clave de la semiótica en España José Romera Castillo, ha continuado brillantemente la labor del Instituto de Semiótica Literaria, Teatral y Nuevas Tecnologías (ISLTYNT), del Departamento de Literatura Española y Teoría de la Literatura de la Facultad de Filología de la UNED desde su fundación en 1991. Desde aquí vaya nuestro agradecimiento por haber contribuido decisivamente para mantener la llama viva de nuestra disciplina en España.

\section{SEMIÓTICA, PANDEMIA, GÉNERO Y ACTUALIDAD}

El monográfico se abre con la intervención del presidente de honor de la AES, José Romera Castillo, quien trata la evolución de las pandemias a lo largo de la historia. Tras revisar casos célebres de la tradición occidental se centra en el tramo final de su trabajo en la incidencia de la COVID-19 en el teatro español, que se vio abocado a plantearse fórmulas para poder subsistir durante los meses que duró el confinamiento. Propuestas como las llevadas a cabo por el Centro Dramático Nacional con el espectáculo La pira, compuesto por La conmoción (Alfredo Sanzol, Victoria Szpunberg y Eva Mir), La distancia (Pau Miró, Juan Mayorga y Andrea Jiménez / Noemí Rodríguez) y La incertidumbre (Pablo Remón, Denise Despeyroux y Lucía Carballal), dan buena cuenta de que la creatividad escénica siempre acaba desbordando sus propias limitaciones para lograr resistir, en este caso pasando del convivio teatral a la virtualidad.

Continúa el monográfico con la contribución de Darío Villanueva, exdirector de la RAE y miembro histórico de la AES. Plantea cómo la pandemia ha provocado el rescate de léxico que, afortunadamente, había quedado en desuso. Pero también muestra cómo han surgido neologismos que incluso han sido recogidos ya en el DEL. Palabras como

\footnotetext{
${ }^{1}$ Véase: http://www.aesemiotica.es/ [20/04/2021]

${ }^{2}$ Véase: https://www2.uned.es/centro-investigacion-SELITEN@T/[20/04/2021]
} 
coronavirus o desescalada, ya instaladas en nuestra habla, demuestran la necesidad de términos que designen nuestra realidad de forma certera. Tras unas reflexiones desde la experiencia personal en torno al confinamiento sufrido en 2020, finaliza proponiendo la imagen de la máscara como símbolo de nuestro tiempo centrándose en la representación del dottore plague.

Por su parte, Alfredo Tenoch Cid Jurado, profesor de la Universidad Autónoma Metropolitana Xochimilco (México), reflexiona sobre la intersección entre los tres conceptos (semiótica, relatos y actualidad) que forman el título del seminario y su función en el pensamiento contemporáneo: "ubica su tarea principal dentro de un proceso de comunicación con una intención específica por comunicar, sin perder de vista la huella cognitiva en los destinatarios", poniendo además el foco de atención en la temporalidad inscrita en esta conceptualización. Para su análisis recurre a Eco, Greimas o Lotman, dando buena cuenta de su erudición y conocimiento profundo del funcionamiento de la semiótica.

Continúa el monográfico Charo Lacalle Zalduendo, presidenta de la AES, quien sigue desarrollando su hipótesis, planteada desde postulados feministas: "La ficción televisiva raramente apuesta por una representación original de la mujer dominante e independiente, sino que más bien opta por la reproducción de características asociadas con la masculinidad" (Lacalle Zalduendo y Gómez Morales, 2016: 64-65). En este caso se centra en la representación de la maternidad en los seriales policiacos españoles. Idealizada según la tradición patriarcal, en este tipo de producciones televisivas se subvierte al demostrar la imposibilidad de encarnar a la madre perfecta en la actualidad — híbrido entre la figura de cuidadora y la de profesional- A través del análisis de la serie española Néboa revelará las estrategias discursivas empleadas para desvelar la falacia neoliberal de la madre ideal.

Lucrecia Escudero, por su parte, acomete el análisis de la última campaña política presidencial de Joe Biden partiendo de su tuit "The people of this nation had spoken". En su caso, estudia la propia lógica temporal de los nuevos medios y la política en torno a la actualidad, que define como "un puro artefacto semio-mediático, ligado a la temporalidad, a la forma en que los medios construyen la percepción de este tiempo". A partir de esta idea analiza el desarrollo y resolución de las elecciones presidenciales estadounidenses de 2020, que depusieron a Donald Trump del poder y convirtieron a Biden en el actual presidente de los EE. UU.

Por último, cierra el monográfico Francisco Linares Alés desde rigurosos postulados semióticos y concluyendo la aplicación de ideas provenientes de la sociocrítica de Edmond Cros. Efectúa la exploración del concepto de "semióticas actuales" para destacar la importancia crucial de lo corporal en la elaboración y el impacto de los discursos contemporáneos. Sobre la actualidad, la Semiótica "no solo la objetiva como hecho del discurso y contenido mental, sino que la adopta con cierto propósito", y es el de significar ciertas prácticas y hechos de acuerdo con una temporalidad subjetivizada y mediada a través del cuerpo. 


\section{CODA FINAL: JORGE LOZANO, IN MEMORIAM}

Este monográfico se cerró tras la triste noticia de la pérdida de Jorge Lozano. Presidente de la AES (2014-2017) y fundador en 2008 del Grupo de Estudios de Semiótica de la Cultura (GESC), su labor en el desarrollo de la Semiótica en España es inestimable, habiendo sido uno de sus principales impulsores a lo largo de su dilatada trayectoria académica. Clásicos como Análisis del discurso. Hacia una semiótica de la interacción textual (1982, junto a Cristina Peña-Marín y Gonzalo Abril), o Análisis del discurso histórico (1987), dan buena cuenta de la altura intelectual de nuestro añorado colega, que se ha convertido en una de las más tristes noticias ocasionadas por esta aciaga pandemia. Tras intervenir en el seminario con gran entusiasmo y vehemencia, rasgos definitorios de su personalidad, la enfermedad no le permitió concluir su contribución a este monográfico. Vaya nuestro recuerdo con él y sus familiares.

\section{REFERENCIAS BIBLIOGRÁFICAS}

Lacalle Zalduendo, C. y Gómez Morales, B. (2016). "La representación de las mujeres trabajadoras en la ficción televisiva española”. Comunicar: Revista Científica Iberoamericana de Comunicación y Educación 47, 59-67.

LozANO, J. (1987). El discurso histórico. Madrid: Alianza.

Lozano, J.; Peña-Marín, C. y Abril, G. (1982). Análisis del discurso. Hacia una semiótica de la interacción textual. Madrid: Cátedra.

(c) $\$($ This work is licensed under a Creative Commons AttributionBY NC ND NonCommercial-NoDerivatives 4.0 International (CC BY-NC-ND).

Fecha de recepción: 15/03/2020

Fecha de aceptación: 21/04/2021 